\title{
Frequency of Isolation and Antibiotic Susceptibility of Micro- Organisms Causing Urinary Tract Infections in Children
}

\author{
SAIMA PERVAIZ ${ }^{1}$, SADIA IKRAM ${ }^{2}$, ANILA ERRUM ${ }^{3}$, SAHAR IQBAL ${ }^{4}$, SARA MASSOOD CHEEMA ${ }^{5}$, NIDA JAVED ${ }^{6}$, SABA \\ SHAMIM $^{7}$ \\ 1,3,4,5 Assistant Professor, Pathology Department, Azra Naheed Dental College, Superior University, Lahore. \\ ${ }^{2}$ Associate Professor, Pathology Department, Azra Naheed Medical College, Superior University, Lahore. \\ ${ }^{6}$ Senior Demonstrator, Al-Aleem Medical College, Lahore \\ ${ }^{7}$ Assistant Professor, IMBB, The University of Lahore, Lahore. \\ Correspondence to: Dr. Saima Pervaiz, Email ID: saimapz@hotmail.com
}

\begin{abstract}
Objective: To determine the frequency of urinary tract infections and the spectrum of microorganisms causing UTI and their antibiotics susceptibility pattern in children age between 1 month and 12 years.

Patients and Methods: In this cross-sectional study, 131 patients of suspected UTI, from the department of pediatric medicine were included, from June-2020 to Nov-2020. 5 milliliters of urine sample were collected and sent to the hospital laboratory for determination of UTI. If UTI was confirmed, samples were further processed to determine the pattern of organisms causing UTI and their antibiotic spectrum.

Results: Mean age of the patients included in this study was $6.07 \pm 256$ years. There were $75(57.25 \%)$ male patients, and $56(42.75 \%)$ female patients. Urinary tract infections were diagnosed in $30(22.90 \%)$ patients. E. coli diagnosed in 14 (46.67\%) patients. Resistant strains were diagnosed in $3(10 \%)$ patients. Regarding antibiotic sensitivity, $3^{\text {rd }}$ generation cephalosporins and carbapenems were sensitive to many organisms.

Conclusion: $E$. coli is the predominant uropathogen causing UTIs in children. Third generation cephalosporins are optimal initial choice for management of UTI in children.

Keywords: urinary tract infections, causating micro-organisms, antibiotic sensitivity.
\end{abstract}

\section{INTRODUCTION}

Urinary tract infections (UTI) are now recognized as one of the commonest infectious diseases, $>10 \%$ people experience at least one episode of UTI during their life time. ${ }^{1}$ As many as $2 \%$ of children have been diagnosed with at least one UTI by the age of 10 years. ${ }^{2}$ It may be an infection of upper urinary tract, lower urinary tract, or both. ${ }^{3}$

Low socioeconomic status is the major factor for development of UTI, other reported risk factors are; old age, diabetes, recurrent UTI, neurogenic bladder retention, poor perineal hygiene, and anatomic/functional urinary tract abnormalities. ${ }^{4,5}$ It effects both sexes and all age groups. Boys are more susceptible during the first year of life: thereafter the incidence is greater in girls. Neonates, girls, young women are more susceptible to UTI. ${ }^{6}$ The affected patients may be symptomatic or asymptomatic at presentation; either type of infection if left untreated can upshot serious sequelae. ${ }^{7}$

Several organisms including fungi and viruses are known to cause UTI but bacteria are known to cause more than $95 \%$ of cases of UTI. The vast majority of organisms causing uncomplicated UTI are Gram negative bacillus Escherichia coli, and Enterococci, Staphylococcus Saprophyticus, Klebsiella spp. and Proteus mirabilis. Escherichia coli is the most common prevalent organism that solely responsible for more than $80 \%$ of UTI cases. ${ }^{8}$

An accurate and timely diagnosis of UTI is important to prevent ascent of infection to upper urinary tract and renal failure. As the number of cases of UTI resistant to antibiotics is increasing day by day. The extensive and inappropriate use of antibiotics has resulted in antibiotic resistance in recent years which has become a worldwide problem. $^{9,10}$

This study was aimed to determine the frequency of urinary tract infections and the spectrum of microorganisms causing UTI and their antibiotics susceptibility pattern in children age between 1 month and 12 years.

\section{METHODS}

In this cross-sectional study, we included 131 patients of suspected UTI, from the department of pediatric medicine were included, from June-2020 to Nov-2020. Children of age 1 month to 12 years having fever $>38 \mathrm{C}$ with chills, urgency and hesitancy of urination, burning micturition or dysuria, suprapubic or flank tenderness, Pyuria or evidence of sepsis were included. Children with congenital malformations of urinary tract, chronic kidney disease, surgical procedures done on urinary tract, having urinary catheter in place for longer duration, and children with other chronic diseases that can cause chronic UTI like cerebral palsy, neurogenic bladder, traumatic injury to the bladder, urinary retention as a complication of stroke, or the children taking antibiotics from last 2 weeks were excluded. Informed consent was taken from parent/guardians.

After enrollment, complete history to look for evidence of urinary tract infection like burning micturition, dysuria, urgency, hesitancy and frequency of urination, flank pain, fever with chills and rigors, previous history of urinary tract infection, history of chronic kidney disease, congenital malformations of urinary tract was taken.

5 milliliters of clean catch urine sample were collected in sterile container and processed within 1 hour of collection using standard methods. A sterile $4.0 \mathrm{~mm}$ platinum wire calibrated loop as used which delivers 0.001 $\mathrm{ml}$ of urine. This loop-full urine sample was inoculated on UTI chrome agar, MacConkey agar and blood agar plates and were incubated aerobically at $37^{\circ} \mathrm{C}$ overnight and for 48 hours in negative cases and read to assess growth of significant bacteria. The specimen was considered positive if more than 5 pus cells/HPF are seen on microscopy or 
those with culture concentration of a single organism is at least $10^{5}$ colonies per $\mathrm{ml}$ of urine. Isolates were gram stained. Bacterial identification was based on standard culture and biochemical characteristics of isolates. $E$ coli was identified as medium, pink-to-red colonies and confirmed by positive indole test. Klebsiella pneumonae are large pink-to-mauve colonies, which was confirmed by negative oxidase and indole test. $P$ mirabilis has translucent blue grey appearing colonies testing negative to indole and oxidase and positive to urease. Enterococcus faecalis is identified by small turquoise mucoid which is negative for catalase and positive for bile esculin. Pseudomonas aeruginosa is identified as clear colonies that is positive for oxidase. Gram negative bacteria will be identified by oxidase test and all other tests of API 20E and API NE (API: analytical profile index). Gram positive organisms were identified by corresponding laboratory test like catalase coagulase, CAMP test and esculin agar. Antibiotic susceptibility of organisms were tested by modified Kirby-bauer disc diffusion technique Mueller Hinton medium using antibiotic disc of oxoid (UK) with the minimum inhibitory concentration (MIC). The susceptibility criteria was applied according to CLSI 2010 guidelines. The organism isolated were tested for sensitivity against different antibiotics discs like levofloxacin, ceftriaxone, ciprofloxacin, amikacin, gentamycin, nitrofurantoin, amoxiciline, imipenam, co-trimoxazole, cephazoline, cefixime and pipademic acid by measuring the diameter of zone of inhibition around each antibiotic disc. If the zone of inhibition will be $>18 \mathrm{~mm}$ around any particular antibiotic disc the organism was considered sensitive for that antibiotic and if it was between 14-18 $\mathrm{mm}$ it is moderately or intermediary sensitive and if it was $<14 \mathrm{~mm}$ than the organism is considered resistant against that antibiotic.

All data was entered in SPSS version-25 for analysis. Spectrum of microorganisms and their antibiotics sensitivity pattern was presented in form of frequency and percentage.

\section{RESULTS}

Mean age of the patients was $6.07 \pm 256$ years. There were $75(57.25 \%)$ male patients, and 56 (42.75\%) female patients.

Urinary tract infections were diagnosed in 30 (22.90\%) patients. While remaining 101 (77.10\%) were not diagnosed of having UTI on culture reporting (Figure 3 ).
Regarding spectrum of causating organisms E. coli was the commonest organism diagnosed in 14 (46.67\%) patients, S. Aeruginosa in 4 (13.33\%) patients, Enterococcus Faecalis in $3(10 \%)$ patients, Klebsella Pneumoiea in $3(10 \%)$ patients, Proteus vulgaris in 2 (6.67\%) patients, Staphylococcus saprophyticus 2 (6.67\%) patients, MRSA in 1 (3.33\%) and proteus Spp. in 1 (3.33\%) patients (Figure 1).

Regarding Antibiotic spectrum, For E. Coli levofloxacin was sensitive to $92.9 \%$ patients, Ceftriaxone in 12 (87.5\%), ciprofloxacin and Amikacin in 13 (92.9\%), Gentamycin in 12 (87.5\%) patients. For Pseudomonas Aeruginosa all drugs were found to be $100 \%$ sensitive except ciprofloxacin, Amoxicilin and Co-trimoxazole which shown $75 \%$ sensitivity. For MRSA only Levofloxacin, Ceftriaxone and Imipenam were sensitive. For Staphylococcus Saprophyticus, and Proteus Spp. almost all drugs were sensitive. Detailed spectrum is given in Table 1.

Resistant strains were diagnosed in $3(10 \%)$ patients, out of which $2(6.67 \%)$ were E. Coli and 1 (3.33\%) were MRSA (Table 2).

All data was entered in SPSS version-16 for analysis. Qualitative variables like gender, age groups, UTI, microorganisms, sensitive strain, resistant strain and multi drug resistant strain and was measured in terms of frequency and percentages and was presented through tables and charts. Mean \pm S.D. was calculated for age.

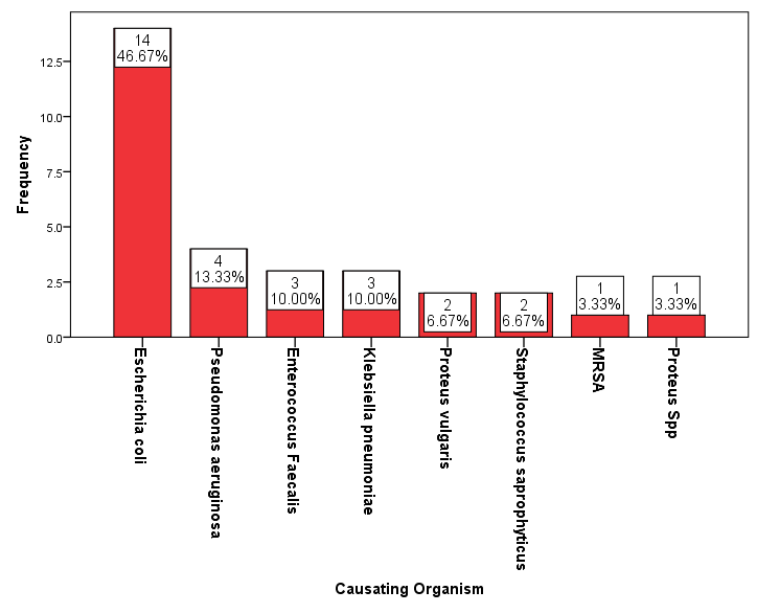

Figure 4. Frequency of Causating Organisms.

Table 1. Antibiotic Susceptibility Patterns of Micro-organisms Causing UTI.

\begin{tabular}{|l|l|l|l|l|l|l|l|}
\hline & E. Coli & $\begin{array}{l}\text { Pseudo. } \\
\text { Aeruginosa }\end{array}$ & E. Faecalis & K. Pneumoniae & $\begin{array}{l}\text { Proteus } \\
\text { Vulgaris }\end{array}$ & $\begin{array}{l}\text { S. Saprophyticus } \\
\text { Spp. }\end{array}$ & MRSA \\
\hline Levofloxacin & $13(92.9 \%)$ & $4(100 \%)$ & $3(10.3 \%)$ & $3(100 \%)$ & $2(100 \%)$ & $2(100 \%)$ \\
\hline Ceftriaxone & $12(87.5 \%)$ & $4(100 \%)$ & $2(66.7 \%)$ & $3(100 \%)$ & $1(50 \%)$ & $2(100 \%)$ \\
\hline Ciprofloxacin & $13(92.9 \%)$ & $3(75 \%)$ & $3(100 \%)$ & $2(66.7 \%)$ & $2(100 \%)$ & $2(100 \%)$ \\
\hline Amikacin & $13(92.9 \%)$ & $4(100 \%)$ & $3(100 \%)$ & $3(100 \%)$ & $2(100 \%)$ & $2(100 \%)$ \\
\hline Gentamycin & $12(85.7 \%)$ & $4(100 \%)$ & $2(66.7 \%)$ & $3(100 \%)$ & $1(50 \%)$ & $2(100 \%)$ \\
\hline Nitrofurantoin & $13(92.9 \%)$ & $4(100 \%)$ & $2(66.7 \%)$ & $2(66.7 \%)$ & $2(100 \%)$ & $2(100 \%)$ \\
\hline Amoxiciline & $10(71.4 \%)$ & $3(75 \%)$ & $3(100 \%)$ & $3(100 \%)$ & $2(100 \%)$ & $2(100 \%)$ \\
\hline Imipenam & $14(100 \%)$ & $4(100 \%)$ & $3(100 \%)$ & $3(100 \%)$ & $2(100 \%)$ & $2(100 \%)$ \\
\hline Cotrimoxazole & $10(71.4 \%)$ & $3(75 \%)$ & $2(66.7 \%)$ & $2(66.7 \%)$ & $2(100 \%)$ & $1(50 \%)$ \\
\hline Cephazoline & $12(85.7 \%)$ & $4(100 \%)$ & $3(100 \%)$ & $3(100 \%)$ & $2(100 \%)$ & $0(100 \%)$ \\
\hline Cefixime & $12(85.7 \%)$ & $4(100 \%)$ & $3(100 \%)$ & $3(100 \%)$ & $1(100 \%)$ & $1(100 \%)$ \\
\hline $\begin{array}{l}\text { Pipademic } \\
\text { Acid }\end{array}$ & $12(85.7 \%)$ & $4(100 \%)$ & $1(33.3 \%)$ & $2(66.7 \%)$ & $2(100 \%)$ & $2(100 \%)$ \\
\hline
\end{tabular}


Table 2. Pattern of Resistance.

\begin{tabular}{|c|c|c|}
\hline & Frequency & Percentage \\
\hline Total Number & 03 & $10 \%$ \\
\hline \multicolumn{3}{|c|}{ Name of Resistant Organisms } \\
\hline E. Coli & 02 & $(6.67 \%)$ \\
\hline$M R S A$ & 01 & $(3.33 \%)$ \\
\hline
\end{tabular}

\section{DISCUSSION}

The pediatric population is at higher risk of infections and UTI is among the commonest causes of morbidity in this population. We found UTI in $22.90 \%$ patients having suspicion of UTI. Mohanty et al. reported UTI in $14.7 \%$ suspected pediatric patients. ${ }^{11}$ Dash et al. and Mehta et al. reported UTI in $34.5 \%$ and $36.6 \%$ patients respectively. ${ }^{12,13}$

A cross sectional study conducted in 2016 in different hospitals of Pakistan showed that overall prevalence of UTI in indoor and outdoor patients is $21.2 \%$ among all age groups out of which $19.4 \%$ were of the age between 0 to 20 year, $49.5 \%$ were of the age falling in the range 21 to 50 years and $31.1 \%$ were above 51 years of age. ${ }^{14}$

In present study, E-Coli was the most common pathogen diagnosed in $46.67 \%$ children, followed by pseudomonas Aeruginosa diagnosed in $13.33 \%$ children. While klebsiella pneumoniae and Enterococcus faecalis was diagnosed in $10 \%$ patients. Woo et al. reported E. Coli prevalence in $86.4 \%$ cases of UTI, Klebsiella spp. in $(4.2 \%)$ cases. While other less common organisms were Enterobacter, Proteus, Enterococcus, Citrobacter, and Staphylococcus. ${ }^{15}$

Another study conducted by Amber et al. on pattern and antibiotic spectrum of UTI in children, reported $E$. coli as the commonest organism diagnosed in $43.2 \%$ patients, P. aeruginosa in $15.5 \%$, Klebsiella pneumoniae in $12.4 \%$, Proteus vulgaris in $5.1 \%$ and Staphylococcus saprophyticus in $3.8 \%$ patients. ${ }^{14}$

Due to rapidly emerging resistance to antibiotics, it is becoming difficult to treat UTI as well. As the children are at higher risk of morbidity and mortality due to infections caused by resistant organisms, the use of inappropriate anti-biotics may lead to adverse prognosis. Therefore, the selection of proper empirical antibiotics is very necessary in these patients. ${ }^{16-18}$

Thus, we investigated the antibiotic susceptibility patterns of E. coli and other pathogens that were isolated in from patients diagnosed with UTI. In present study antibiotic resistance was diagnosed in $10 \%$ patients, of which $6.66 \%$ patients were having $E$. Coli infection and $3.33 \%$ patients were having MRSA.

The presence of resistant strains of UTI to routinely available antibiotics in Pakistani patients is due to different factors. Most important of one the unjudicial prescription of anti-biotics, and almost 80 to $90 \%$ antibiotics are available over the counter and no prescription is required to get these from pharmacies. Moreover, $3^{\text {rd }}$ general antibiotics are is common practice to treat less virulent organisms such as those causing pharyngitis or otitis. The other reasons are unavailability of infection control departments and policies in our hospitals. ${ }^{19-21}$

\section{CONCLUSION}

E. coli is the predominant uropathogen causing UTIs in children. Most of the strains causing UTI are resistant to first general antibiotics. Third generation cephalosporins are optimal initial choice for management of UTI in children.

\section{REFERENCES}

1. Akhtar N, Rahman R, Sultana S. Antimicrobial sensitivity pattern of Escherichia coli causing urinary tract infection in Bangladeshi patients. American Journal of Microbiological Research. 2016;4(4):122-5.

2. Moore CE, Sona S, Poda S, Putchhat H, Kumar V, Sopheary S, et al. Antimicrobial susceptibility of uropathogens isolated from Cambodian children. Paediatrics and international child health. 2016;36(2):113-7.

3. Rezaee MA, Abdinia B. Etiology and Antimicrobial Susceptibility Pattern of Pathogenic Bacteria in Children Subjected to UTI: A Referral Hospital-Based Study in Northwest of Iran. Medicine (Baltimore). 2015;94(39):e1606.

4. Gondim R, Azevedo R, Braga AANM, Veiga ML, Barroso U, Jr. Risk factors for urinary tract infection in children with urinary urgency. Int Braz J Urol. 2018;44(2):378-83.

5. Shiralizadeh S, Taghizadeh S, Asgharzadeh M, Shokouhi B, Gholizadeh $\mathrm{P}$, Rahbar $\mathrm{M}$, et al. Urinary tract infections: raising problem in developing countries. Rev Med Microbiol. 2018;29(4):15965.

6. Schmidt B, Copp HL. Work-up of pediatric urinary tract infection. Urol Clin. 2015;42(4):519-26.

7. Flores-Mireles AL, Walker JN, Caparon M, Hultgren SJ. Urinary tract infections: epidemiology, mechanisms of infection and treatment options. Nat Rev Microbiol. 2015;13(5):269-84.

8. Klein RD, Hultgren SJ. Urinary tract infections: microbial pathogenesis, host-pathogen interactions and new treatment strategies. Nat Rev Microbiol. 2020;18(4):211-26.

9. Edlin RS, Copp HL. Antibiotic resistance in pediatric urology. Ther Adv Urol. 2014;6(2):54-61.

10. Vazouras K, Velali K, Tassiou I, Anastasiou-Katsiardani A, Athanasopoulou K, Barbouni A, et al. Antibiotic treatment and antimicrobial resistance in children with urinary tract infections. J Glob Antimicrob Resist. 2020;20:4-10.

11. Mohanty S, Kapil A, Das BK, Dhawan B. Antimicrobial resistance profile of nosocomial uropathogens in a tertiary care hospital. Indian J Med Sci. 2003;57(4):148-54.

12. Dash M, Padhi S, Mohanty I, Panda P, Parida B. Antimicrobial resistance in pathogens causing urinary tract infections in a rural community of Odisha, India. J Family Community Med. 2013;20(1):20-6.

13. Mehta M, Bhardwaj S, Sharma J. Screening of urinary isolates for the prevalence and antimicrobial susceptibility of Enterobacteria other than Escherichia coli. Int J Life Sci Pharma Res. 2013;3(1):100-4.

14. Amber M, Shoaib M, Rehman A, Zafar MM, til Ain Q, Naseer F, et al. Comparative study of Causative agents of UTI among Indoor, outdoor, Children and Adult Patients of Pakistani Population. Bull Env Pharmacol Life Sci. 2016;5(8):56-63.

15. Woo B, Jung Y, Kim HS. Antibiotic Sensitivity Patterns in Children with Urinary Tract Infection: Retrospective Study Over 8 Years in a Single Center. Childhood Kidney Dis. 2019;23(1):22-8.

16. Najeeb S, Tariq N, Kanwal M, Sadiq MU, Sadiq MI. Antibiotic Sensitivity Patterns of Uropathogens in Children: The Current Trend. J Islamabad Med Dent Coll. 2019;8(3):101-6.

17. Mahony M, McMullan B, Brown J, Kennedy SE. Multidrug-resistant organisms in urinary tract infections in children. Pediatr Nephrol. 2020;35(9):1563-73.

18. Chen H-E, Tain Y-L, Kuo H-C, Hsu C-N. Trends in Antimicrobial Susceptibility of Escherichia coli Isolates in a Taiwanese Child Cohort with Urinary Tract Infections between 2004 and 2018. Antibiotics. 2020;9(8):501.

19. Ali M, Irtiqa A, Mahrukh F, Tooba A. Factors Leading to Acquired Bacterial Resistance Due to Antibiotics in Pakistan. Curr Tr Biotech \& Microbio. 2018;1(1):000101.

20. Mohsin M. The under reported issue of antibiotic-resistance in foodproducing animals in Pakistan. Pak Vet J. 2019;39(3).

21. Faizullah M, Rahman N, Umar M, Anwar M, Sarfraz M. A crosssectional study on knowledge, attitude and practices of medical doctors towards antibiotic prescribing patterns and resistance in Khyber Pakhtun Khawah, Pakistan. J Appl Pharm Sci. 2017;7(12):3846. 